\title{
Rayonnement ultraviolet et santé
}

\author{
J.P. CÉSARINI ${ }^{1}$
}

(Manuscrit reçu le 11 mai 2007, accepté le 2 juillet 2007)

RÉSUMÉ Le rayonnement ultraviolet est responsable d'un large impact sur les structures biologiques. C'est un carcinogène bien documenté. Alors qu'un certain nombre d'effets positifs peut être associé à ce rayonnement, les surexpositions induisent des effets aigus et chroniques sur la peau, les yeux et le système immunitaire. Les effets négatifs associés aux expositions peuvent être largement évités. Réduire les expositions au rayonnement UV par des modifications du comportement et de l'environnement constitue le but principal des mesures préventives. Longtemps considéré comme «innocent", le rayonnement UVA possède des propriétés mutagènes et carcinogènes qui sont aujourd'hui bien documentées et leurs mécanismes de production semblent aujourd'hui élucidés et en particulier la photochimie des UVA solaires dans la peau. Aujourd'hui, les mécanismes de la photocarcinogenèse induite par les UVA ou par les UVB paraissent identiques, leur importance étant égale dans le rayonnement solaire. Les efforts de prévention doivent s'adresser en priorité aux enfants mais également dans le cadre du travail, en particulier en extérieur. Des guides pratiques sont fournis par l'OMS pour aider les autorités sanitaires dans la mise en application d'une politique de prévention afin de réduire les risques associés au rayonnement ultraviolet.

ABSTRACT Ultraviolet radiation and health.

Ultraviolet radiation is a well-known carcinogen that has a large impacy on biological structures and as a consequence on human health. While they have some positive effects associated with exposure to ultraviolet radiation, over exposures can lead to acute and chronic health effects on skin, eyes and immune system. With the right preventable measures, the negative effects associated with exposure to ultraviolet radiation can be largely avoided. The principal goal of preventive efforts is to reduce exposure to ultraviolet radiation through behavioral and environmental changes. While the mutagenic and carcinogenic properties of UVA are well established, mechanisms of UVA mutagenesis remain a matter of debate. However, the role of UVA in solar photocarcinogenesis appears more prominent that it was thought before. The most recent results have shown that mechanisms of photocarcinogenesis induced either by UVA or UVB are identical, their weight in the solar radiation being equivalent. Preventive efforts should be directed toward children and toward outdoor workers. Practical guides are provided by WHO to assist local authorities to implement policies, practices and environmental changes that will reduce the risks associated with ultraviolet radiation.

Keywords: ultraviolet radiation / health / carcinogenesis / prevention / standards

\footnotetext{
1 SFRP, Section rayonnements non-ionisants, 83 avenue Simon Bolivar, 75019 Paris, France.
} 
J.P. CÉSARINI

\section{Introduction}

Le rayonnement ultraviolet (RUV) se positionne dans la portion extrême des radiations non-ionisantes $\mathrm{du}$ spectre électromagnétique, au contact $\mathrm{du}$ rayonnement $\mathrm{X}$. Le mode primaire d'interaction avec la matière est différent de celui produit par ionisation. Présent dans l'émission solaire, le RUV n'est pas perçu par les récepteurs rétiniens, cônes et bâtonnets n'y étant pas sensibles. Aucune sensation de chaleur n'est perçue par les récepteurs cutanés. L'absence de perception immédiate de ce rayonnement relativement énergétique en fait le danger immédiat et à long terme par accumulation des lésions induites dans le génome cellulaire des tissus atteints : tissu cutané et tissu oculaire.

\section{Rappel de notions élémentaires}

\subsection{Données physiques}

Le rayonnement ultraviolet est compris entre $100 \mathrm{~nm}$ (énergie photonique de $12 \mathrm{eV}$ ) et $400 \mathrm{~nm}$ (énergie photonique de $3 \mathrm{eV}$ ). On distingue les UVC, 100-280 nm (l'ozone stratosphérique absorbe les UVC et une grande partie des UVB avant que le rayonnement solaire atteigne la terre) - les UVB, 280-315 nm (on utilise fréquemment $320 \mathrm{~nm}$ pour séparer UVA et UVB) - les UVA, 315-400 nm.

\subsubsection{Le rayonnement solaire global}

Le rayonnement solaire global présente des variations en fonction de l'angle zénithal (hauteur du soleil sur l'horizon), c'est-à-dire en fonction de l'heure, de la saison, de la latitude. La composition du spectre solaire varie en fonction de l'épaisseur de la couche atmosphérique traversée. En pratique, l'UVB est absorbé pratiquement totalement quand le soleil est en dessous de $45^{\circ}$ par rapport à l'horizon (règle pratique de l'ombre portée : le risque lié au rayonnement UVB est significatif quand l'ombre portée est plus courte que la taille du sujet en position verticale).

L'altitude est également un facteur important, une augmentation de $4 \%$ du rayonnement UVB par tranches de 300 m étant observée en général. La couverture nuageuse est également un facteur important de variables, nature et altitude de l'ennuagement sont capables soit de supprimer complètement le RUV, soit, plus rarement, d'augmenter le RUV à la surface terrestre.

Enfin, la réflexion sur les surfaces peut amplifier significativement le rayonnement direct : neige et glace, surf costal, reflètent jusqu'à $80 \%$ du RUV, 
sable ou ciment environ $15 \%$, la verdure étant faiblement réflecteur $4 \%$ (WHO/ UNEP/ICNIRP, 1994).

\subsubsection{Les sources $U$ V artificielles}

Les applications industrielles ou commerciales du rayonnement ultraviolet artificiel sont nombreuses. La conception de ces sources interdit en principe toute exposition de leurs manipulateurs. Néanmoins, par défaut ou par erreur de manipulation, des accidents sont toujours possibles. Les mesures doivent être prises dans le cadre de la sécurité pour éviter les surexpositions aiguës aussi bien que l'accumulation des expositions.

Le rayonnement ultraviolet peut être considéré comme un polluant de certaines sources d'éclairage (halogènes, tubes fluorescents) ou de certaines technologies telles que les arcs électriques nécessaires aux opérations de soudure. Les UVC ou les UVA sont en fait utilisés industriellement pour leur propriété de stérilisation, de polymérisation des encres et des peintures, d'excitation de la fluorescence de certains composés chimiques ( $c f$. Tab. I). Dans tous les cas, la protection des utilisateurs doit être une priorité.

\subsection{Pénétration du rayonnement ultraviolet dans les tissus}

\subsubsection{Pénétration dans la peau}

Le rayonnement ultraviolet incident sur le tégument subit des phénomènes de réflexion réfraction ou absorption, dont l'importance et la nature sont fonctions de la longueur d'onde.

Les UVC ne pénètrent pas en dessous de la couche cornée où ils sont entièrement absorbés. Seuls $10 \%$ de l'UVB incident atteignent la couche basale où se localisent les kératinocytes en division (toutes les 3 semaines) et les mélanocytes producteurs de mélanine. Les capillaires situés entre les crêtes papillaires peuvent être atteints. L'épiderme absorbe $50 \%$ du rayonnement UVA qui pénètre jusqu'aux vaisseaux et aux fibres du derme.

\subsubsection{Pénétration dans l'œeil}

La complexité des tissus oculaires (cornée, chambre aqueuse, cristallin, vitrée) assure une bonne protection vis-à-vis de la pénétration des RUV puisque ceux-ci, en conditions normales, n'atteignent pas la rétine. Cependant, le risque d'atteinte rétinienne chez l'enfant est bien réel car le cristallin, encore vierge de pigments d'usure, n'absorbe pas les UV, ce qui justifie une protection spécifique. 
TABLEAU I

Le rayonnement ultraviolet et ses applications industrielles ou commerciales. The ultraviolet radiation and its industrial or commercial applications.

\begin{tabular}{|c|c|c|c|}
\hline Domaine industriel & Applications & Lampes & Émissions utiles \\
\hline Imprimerie & Polymérisation encre & $\begin{array}{l}\mathrm{Hg} \text { haute pression } \\
\text { Métalloïdes }\end{array}$ & $\begin{array}{l}\text { UVA, B, C } \\
\text { UVA }\end{array}$ \\
\hline Imprimerie & Séchage encre & Incandescence & IR \\
\hline Photolithographie & Plaque & $\begin{array}{l}\text { Xe haute pression } \\
\text { Métalloïdes } \\
\text { Tungstène halogène }\end{array}$ & $\begin{array}{l}\text { UVA, visible } \\
\text { UVA } \\
\text { UVA, visible }\end{array}$ \\
\hline $\begin{array}{l}\text { Photocopie } \\
\text { (Système diazo) }\end{array}$ & Expositions & $\begin{array}{l}\text { Fluorescence } \\
\mathrm{Hg} \text { haute pression }\end{array}$ & $\begin{array}{l}\text { UVA, bleu } \\
\text { UVA }\end{array}$ \\
\hline $\begin{array}{l}\text { Photocopie } \\
\text { (Papier/ZnO) }\end{array}$ & $\begin{array}{l}\text { Expositions } \\
\text { Séchage }\end{array}$ & $\begin{array}{l}\text { Fluorescence } \\
\text { Tungstène halogène }\end{array}$ & $\begin{array}{l}\text { Bleu, vert } \\
\text { Visible \& IR }\end{array}$ \\
\hline Peintures & Polymérisation & $\mathrm{Hg}$ haute pression & UVA, UVB, UVC \\
\hline Peintures & Séchage & $\begin{array}{l}\text { Incandescence } \\
\text { Halogène }\end{array}$ & $\begin{array}{l}\text { IR } \\
\text { IR }\end{array}$ \\
\hline Semi-conducteurs & Exposition & $\mathrm{Hg}$ haute pression & UVA \\
\hline Circuits imprimés & Exposition & $\begin{array}{l}\mathrm{Hg} \text { haute pression } \\
\text { Fluorescence }\end{array}$ & $\begin{array}{l}\text { UVA } \\
\text { UVA }\end{array}$ \\
\hline $\begin{array}{l}\text { Réactions chimiques } \\
\text { (Photo-oxydation) }\end{array}$ & Réacteur photochimique & $\begin{array}{l}\mathrm{Hg} \text { haute pression } \\
\text { Métallö̈des }\end{array}$ & $\begin{array}{l}\text { UVA } \\
\text { UVA, visible }\end{array}$ \\
\hline Soudures & Soudure à l'arc & Électricité & UVA, UVB, UVC, visible \\
\hline Technologie du vide & Plasma & Arc xénon & UVA, UVB, UVC, visible \\
\hline Désinfection & Surfaces, air, eau & $\mathrm{Hg}$ haute pression & UVA, UVB, UVC \\
\hline Hygiène alimentaire & Attractant d'insectes & Fluorescence & UVA \\
\hline Analyses & $\begin{array}{l}\text { Spectrométrie } \\
\text { Recherche génome }\end{array}$ & $\begin{array}{l}\mathrm{Hg} \text { haute pression } \\
\text { Fluorescence }\end{array}$ & $\begin{array}{l}\text { UVA, UVB, UVC } \\
\text { UVA }\end{array}$ \\
\hline Dermatologie & Traitements médicaux & $\begin{array}{l}\mathrm{Hg} \text { haute pression } \\
\mathrm{Hg} \text { basse pression }\end{array}$ & UVA, UVB \\
\hline
\end{tabular}

\subsection{Effets biologiques}

Acides nucléiques, lipides et protéines, absorbent directement les UVC dont l'énergie est ainsi transférée aux constituants biologiques. Il en est de même pour les UVB. Les UVA sont plus particulièrement absorbés par certains chromophores et leur énergie est transférée aux autres constituants cellulaires pour des réactions indirectes de photosensibilisation et activation des espèces réactives de l'oxygène.

La cible principale du RUV est le matériel nucléaire génétique où par action directe, les UVC et UVB entraînent la production de dimères de pyrimidine (entre deux bases pyrimidiques adjacentes), de type cyclobutane (5-6), ou 
pyrimidine-pyrimidone (6-4). Les dimères de cytosine (entre deux bases cytosine adjacentes) auront pour conséquence des mutations tandem CC $\rightarrow$ TT qui constitue une signature de l'agression UV. UVB et UVA produisent par réaction indirecte (réaction de radicaux hydroxyles $\mathrm{OH}^{\circ}$ ), l'oxydation de bases puriques et produisent des 8 oxo-guanines. Certaines lésions de l'ADN entraînent la rupture d'une ou des deux branches de l'hélice avec des conséquences importantes sur la traduction des messages génétiques.

Les lésions induites par les RUV sont plus ou moins rapidement réparées par des systèmes réparateurs enzymatiques complexes, les réparations pouvant s'effectuer sans erreurs ou avec erreurs dont les conséquences, dans les cellules souches, conduisent aux mutations et éventuellement, au processus de cancérisation.

Chez l'homme, la capacité de réparation diminue avec l'âge mais il existe également une variabilité individuelle de la capacité de réparation génétiquement définie. Ceci s'exprime par la notion de «capital solaire». Les relations significatives entre phototoxicité, photomutagénicité et photocarcinogenèse ont été largement démontrées.

Le gène p53 est la cible élective de la carcinogenèse induite par le RUV. Ce gène code pour une protéine qui contrôle les divisions cellulaires. Les RUVs, comme les radiations ionisantes ou certaines substances génotoxiques, induisent des lésions de l'ADN, activatrices de p53 qui, à son tour, induit l'arrêt du cycle cellulaire en phase G1, permettant à la cellule, soit de réparer les lésions avant la réplication, soit d'entrer en apoptose si les dommages sont trop importants. Dans les kératinocytes, une mutation induite dans un gène p53 par les RUV, favorise l'expansion clonale de ces kératinocytes réalisant ainsi des kératoses actiniques. Une exposition ultérieure aux RUV induit une seconde mutation sur le brin sain et, dès lors, la multiplication kératinocytaire devient anarchique : c'est l'épithélioma spinocellulaire. Quel que soit le système expérimental utilisé et dans les cancers cutanés humains, les doubles mutations de p53 surviennent essentiellement sur les mêmes zones de fragilité et se traduisent par des transitions spécifiques du rayonnement ultraviolet.

\subsection{Traduction clinique des effets biologiques}

\subsubsection{L'érythème actinique}

L'exposition de la peau au RUV, pour une dose suffisante (15 à 30 minutes d'exposition solaire, à 13 heures par ciel clair sur les bords de la Méditerranée) déclenche les processus complexes (production de cytokines, libération de 
fragments d'ADN lésé) conduisant au classique érythème actinique (coup de soleil) quelques heures après l'exposition et à l'activation de la tyrosinase mélanocytaire qui augmente la production de mélanine, induisant à une hyperpigmentation (bronzage) quelques jours après l'exposition. L'érythème actinique traduit les signes d'une inflammation : rougeur, chaleur, et sensation de brûlure plus ou moins intense.

Le spectre solaire dans l'UV est un spectre continu et chaque longueur d'onde présente une efficacité relative pour déclencher l'érythème. Ceci a permis de définir un spectre d'efficacité érythémale qui a fait l'objet d'une normalisation (ISO/CIE, 1998) ainsi qu' une standardisation de la dose érythémale efficace $\left(S E D=100 \mathrm{~J} \mathrm{~m}^{-2}\right)$,

\subsubsection{Sensibilité aux radiations $U V$ et phototypes}

L'inégalité des réactions aux expositions RUV est bien connue. La sensibilité à l'érythème actinique dépend de plusieurs facteurs dont les plus importants sont la quantité et la qualité de mélanine d'une part et l'épaisseur de l'épiderme d'autre part. Cette sensibilité est à l'origine d'une classification des peaux humaines en fonction de leur type et charge en mélanine et de leur adaptation aux expositions répétées. C'est ainsi que l'on distingue les sujets mélano-compromis qui brûlent facilement et bronzent pas ou peu, les sujets mélano-compétents qui, éventuellement brûlent mais bronzent bien et les sujets mélano-protégés qui ne brûlent qu'exceptionnellement et présentent rapidement une peau très sombre. Cette classification est aujourd'hui retenue par l'OMS pour la communication sur les risques liés aux expositions aux RUV : l'épidémiologie des cancers cutanés a montré que $90 \%$ de ceux-ci sont observés chez les sujets mélano-compromis.

\subsubsection{Phototoxicité, photoallergie et photoréacteurs chroniques}

La présence de molécules endogènes ou exogènes dans l'épiderme est susceptible d'induire des phénomènes de phototoxicité (érythème actinique pour des doses nettement inférieures à la dose érythémale du phototype) et des phénomènes de photoallergie où le phénomène phototoxique est amplifié par la réaction immunitaire dirigée contre le photo-allergène.

\subsubsection{Adaptation aux expositions $U V$ répétées et hyperpigmentations séquellaires}

La répétition aux expositions UV induit une adaptation qui se traduit par la néomélanogenèse et l'épaississement de l'épiderme (doublement). Ce dernier phénomène a pour effet de soustraire la couche basale à l'action directe des UVB et d'une grande partie des UVA réduisant ainsi les dommages aux couches les plus 
profondes de l'épiderme et du derme. Cependant, l'arrêt de stimulation induit un état de steady state atteint généralement après 3 semaines d'exposition. En absence de nouvelles expositions, le retour à la normale s'effectue en trois mois.

En cas d'exposition très importante (10 fois la dose érythémale minimale), une brûlure du second degré est induite, s'accompagnant d'une altération définitive des mélanocytes qui, soit sont détruits (hypopigmentation), soit multipliés (taches hyperpigmentées ou lentigines solaires définitives).

\subsubsection{Immunodépression cutanée}

Les cellules de Langerhans, normalement situées sous la couche cornée, sont intensément agressées par les RUV B et A directement au niveau de leur structure, soit indirectement par l'acide urocanique trans. Leur disparition partielle et leurs fonctions altérées (présentation des antigènes d'origine exogène ou endogène) réduit considérablement les défenses immunitaires $(50 \%$ pour un érythème actinique léger) et cela durablement (15 jours après une irradiation unique). La traduction de ce phénomène peu apparent peut avoir des conséquences importantes : herpes, mycoses estivales, accélération de la carcinogenèse).

\subsubsection{Le vieillissement cutané photo-induit ou héliodermatose}

L'accumulation des désordres créés par des expositions répétées aux RUV se traduit par un vieillissement dont les aspects sont spécifiques quand ils sont comparés au vieillissement «chronologique». L'épiderme est aminci, dyschromique, le tissu de soutien (collagène et élastique) est remplacé par un matériel peu structuré qui favorise la dilatation des capillaires se traduisant par des télangiectasies. Des rides plus ou moins profondes apparaissent (cou de marin).

\subsubsection{Les cancers cutanés}

Ils représentent la conséquence la plus redoutable des expositions répétées aux RUV. Ils sont exceptionnellement constatés avant l'âge de 40 ans (sauf pour les sensibilités particulières et les conditions extrêmes d'exposition).

Les cancers cutanés non-mélaniques, en France, représentent environ 70000 cas par an, dont 50000 sont des épithéliomas baso-cellulaires et 20000 des épithéliomas spino-cellulaires. Leur incidence double tous les 20 ans.

\subsubsection{1. Épithéliomas spino-cellulaires}

On peut les définir comme la prolifération incontrôlée des kératinocytes de la basale épidermique. Ils sont précédés par des états précancéreux (kératoses 
actiniques non invasives). D'évolution lente, ils métastasent rarement malgré l'invasion du derme. Certaines localisations sont cependant de mauvais pronostic (lèvres, conjonctive). Le risque de survenue est augmenté avec l'âge, essentiellement chez les sujets mélano-compromis. Ce risque est augmenté par les coups de soleil éprouvés dans l'enfance et par le cumul des doses annuelles. Ils surviennent essentiellement sur les zones d'exposition permanente.

\subsubsection{2. Épithéliomas baso-cellulaires}

Reproduisant par leurs inflexions morphologiques les différentes cellules potentiellement développées à partir des cellules germinales épidermiques, ce sont des proliférations classiquement peu invasives et non métastasiantes. Ils seraient plus particulièrement liés à l'action des UVA. Ils surviennent chez les sujets présentant les mêmes facteurs de risques que les épithéliomas spinocellulaires mais sont présents également en zones d'exposition intermittentes (tronc, et racines des membres). Ils surviennent également chez les sujets mélanocompétents mais ne semblent pas alors liés aux doses annuelles cumulées.

\subsubsection{Mélanomes malins}

Ils sont le résultat de la prolifération des mélanocytes. Leur étiologie n'est certainement pas univoque car leur morphologie, leurs aspects cliniques et histopathologiques permettent de reconnaître différentes formes. On distingue les lentigos malins qui sont des taches pigmentées irrégulières s'étalant horizontalement et pendant plusieurs années sans caractère invasif. Leur potentiel est faiblement métastatique et leurs caractéristiques épidémiologiques les font relier à la multiplication des expositions au rayonnement UV de faible dose sur les surfaces habituellement exposées à la lumière (face, avant-bras, dos des mains). Les mélanomes à extension superficielle (caractérisés par une phase horizontale longue) et les mélanomes nodulaires (caractérisés par une phase horizontale brève ou absente) sont des tumeurs pigmentées de caractère invasif et métastatique, entraînant une issue fatale en absence de traitement chirurgical précoce. La profondeur atteinte (invasion vasculaire) détermine leur pronostic.

Ils représentent 8000 cas par an en France et sont en augmentation annuelle de $7 \%$ depuis le milieu du $20^{\mathrm{e}}$ siècle. Leur incidence selon le sexe et l'âge est variable selon les pays avec toutefois une prédominance du sexe féminin. Leur incidence double tous les 15 ans mais un fléchissement de l'augmentation semble perceptible depuis que les campagnes de photoprotection et de diagnostic précoce ont été engagées. Leur distribution géographique montre un gradient proportionnel à la latitude et à la proximité de la mer. 
L'étude des populations migrantes a montré que le risque est d'autant plus important que les fortes expositions se produisent avant l'âge de 10 ans. En général, les travailleurs « col blanc » présentent plus de mélanomes que les travailleurs « en extérieur », ce qui souligne le rôle des surexpositions intermittentes.

\subsubsection{Les cataractes}

Les cataractes (opacification progressive du cristallin) constituent un sérieux problème de santé publique. Contrairement aux conséquences cutanées des expositions UV, elles atteignent toutes les populations et frappent environ 200000 personnes par an en France. Leur traitement (chirurgie et prothèse) constitue un coût financier conséquent. La dégénérescence maculaire liée à l'âge serait pour certains la conséquence de l'accumulation d'agression par la partie bleue du spectre visible. Plus que jamais, la prévention de ces pathologies de plus en plus invalidantes, dans une population vieillissante, devrait constituer une priorité de santé publique.

\subsection{Conséquences des expositions aux RUV pour la santé}

L'OMS, le CIRC, la FDA ont officiellement classé le rayonnement UV comme carcinogène. Ceci conditionne désormais les recommandations de santé publique en général avec un abord spécifique dans le cadre du travail. Au niveau des Communautés européennes le risque UV a été pris en compte, suivant les recommandations de l'ICNIRP et de l'ACGIH (USA), dans la directive « basse tension » (Directives du Parlement Européen, $19^{\mathrm{e}}$ directive, 2006). La métrologie du rayonnement a fait l'objet d'une série de normes (Norme NF EN 14255-1 à 4, 2005, 2006, 2007).

Actuellement, il y a débat sur l'utilité des expositions UV (essentiellement UVB) vis-à-vis de la production de vitamine D dont les besoins chez l'enfant et chez les seniors sont augmentés. Les populations à peau sombre vivant au-delà des latitudes méditerranéennes pourraient souffrir de carence en cas de déficit d'apport nutritionnel. Cette problématique sera plus facilement réglée par l'apport alimentaire de vitamine D que par la recommandation d'expositions aux RUV.

\section{Quelques actualités remarquables}

\subsection{Réévaluation de la photochimie de l'UVA solaire dans la peau humaine}

Les propriétés mutagènes et carcinogènes des UVA, comme des UVB, sont aujourd'hui bien établies. Le spectre d'efficacité des mutations induites dans les 
fibroblastes humains a été défini. Comme pour les UVB, les transitions $\mathrm{C} \rightarrow \mathrm{T}$ forment la majorité des mutations UVA. De même, on retrouve des points chauds identiques (pyrimidines) sur les brins non transcrits. On a identifié également des mutations en tandem CC $\rightarrow$ TT. Cependant, les UVA activent moins p53, ce qui peut expliquer un potentiel mutagène différent (Kappes et al., 2006).

Grâce à l'HPLC couplée à la spectrométrie de masse, l'équipe de Cadet a montré, sur des kératinocytes et la peau humaine complète, que les dimères de pyrimidine étaient produits en quantités importantes, soit directement par les UVB, soit indirectement par les UVA. Les UVA, par un mécanisme oxydatif indirect, produisent des 8-oxoguanines en quantités plus faibles que les dimères. Ces derniers sont cependant réparés plus lentement que ceux induits par les UVB. L'épaisseur de la peau complète protège mieux contre l'agression par les UVB que contre l'agression par les UVA. Il convient d'en tenir compte dans les stratégies de photoprotection par les produits antisolaires ainsi que dans l'approche législative vis-à-vis des risques présentés par les solariums (UVA) (Mouret et al., 2006).

L'éditorial du PNAS (Mitchel, 2006) souligne les conséquences de ces récentes découvertes et insiste sur la nécessité d'une protection additionnelle sur toute l'étendue du spectre solaire et en particulier vis-à-vis des UVA. Il ne faut pas protéger uniquement contre les UVB (les SPF des préparations cosmétiques sont basés sur l'érythème) mais également introduire la notion de ratio UVB/UVA dans la photoprotection. La dangerosité de la pratique du bronzage par UV artificiels spécifiquement UVA est soulignée : «Il n’y a pas de bons UV ».

\subsection{Réévaluation des spectres d'action du risque ultraviolet}

Le spectre d'action $S(\lambda)$, «fonction du risque $U V$ », est utilisé pour évaluer le risque des sources UV depuis plus de 30 ans dans différentes normes (ACGIH, ICNIRP). Les risques d'effets aigus pour la cornée et la peau, étendus aux effets chroniques, sont évalués grâce à ce spectre d'efficacité obtenu avec des largeurs de bandes spectrales importantes (+de $10 \mathrm{~nm}$ ). Les progrès techniques permettent des largeurs de bandes de $1 \mathrm{~nm}$ et les précisions ainsi obtenues ne modifient pas substantiellement le spectre d'efficacité. La «fonction du risque UV $S(\lambda)$ » reste inchangée (Chaney et Sliney, 2005).

\subsection{Photoproduction de vitamine $D$ et photodégradation d'acide folique : la controverse}

Une large part des besoins en vitamine D est couverte par la nutrition, mais pour les populations à peau claire, l'épiderme synthétise une part non négligeable de la vitamine D à partir du cholestérol sous l'action essentiellement des UVB. 
La prolifération cellulaire étant synchronisée grâce aux récepteurs de la vitamine $\mathrm{D}$, pour certains, un apport supplémentaire de vitamine $\mathrm{D}$ permettrait de réduire l'incidence des cancers et de certaines pathologies. Ceci conduirait à une promotion des expositions volontaires. Cependant, l'acide folique circulant est détruit par les UVB atteignant les capillaires sous-épidermiques. Le déficit en acide folique est associé à des anomalies du tube neural chez l'embryon et à une réduction de la fertilité chez le mâle. Une réduction de la photoprotection (antiUVB) et les surexpositions volontaires aux UVB ne sont pas justifiées.

\subsection{Les enfants et le soleil}

Toutes les enquêtes épidémiologiques montrent que les surexpositions solaires survenant avant la puberté induisent la survenue plus précoce et plus fréquente d'un certain nombre de pathologies, en particulier, les mélanomes malins.

La réaction érythémateuse cutanée chez l'enfant, pour des raisons anatomophysiologiques, est moins marquée que chez l'adulte, cependant, les dégâts cellulaires sont plus importants. La photoprotection cutanée doit être renforcée surtout avant la puberté (l'usage de vêtement doit être préféré aux crèmes antisolaires).

Avant l'âge de 7 ans, la transparence du cristallin permet aux UV d'atteindre la rétine. Les yeux doivent être particulièrement protégés (visière, lunettes) (Zuurbier et al., 2007).

\subsection{La communication du risque $U V$ pour le public}

Selon les recommandations de l'OMS, l'information du public sur les risques liés aux surexpositions solaires, doit se faire de manière alarmiste grâce à une meilleure appréciation et information du rayonnement solaire. L'index UV a été développé pour favoriser cette prise de connaissance et sa communication doit s'accompagner de messages simples permettant de protéger efficacement les zones exposées et les yeux quotidiennement et pendant les vacances (Tab. II). L'index UV est défini par la formule : $\mathrm{I}_{\mathrm{UV}}=40 \times \mathrm{I}_{\mathrm{eff}}\left(\mathrm{W} \mathrm{m}^{-2}\right)$ (WHO/WMO/ UNEP/ICNIRP, 2002).

On peut consulter les informations concernant l'index-UV et les conditions quotidiennes d'exposition et de photoprotection sur Internet www.soleil.info.

Les sources d'éclairage destinées à émettre un rayonnement visible uniquement sont en générale bien contrôlées et leur émission UV pratiquement éradiquée (ICNIRP, 1997 ; CIE, 2002 ; Norme NF/EN 13032-1, 2004). 
TABLEAU II

Corrélations entre index-UV et photoprotection.

Correlations between UV index and photoprotection.

\begin{tabular}{llll}
\hline categories d'exposition & index-UV & photoprotection & commentaires \\
\hline Faible & $1 \& 2$ & Non & $\begin{array}{l}\text { Danger négligeable } \\
\text { Modérée }\end{array}$ \\
$\begin{array}{lll}\text { Élevée } \\
\text { Très élevée }\end{array}$ & $8 \& 7$ à 10 & Nécessaire & $\begin{array}{l}\text { Porter une chemise et un chapeau, appliquer un } \\
\text { écran solaire. } \\
\text { Porter des lunettes CE3 }\end{array}$ \\
Extrême & 11 et + & Renforcée & $\begin{array}{l}\text { Éviter l'extérieur pendant les heures chaudes et } \\
\text { rechercher l'ombre. Tous les systèmes de } \\
\text { photoprotection doivent être mis en œuvre. } \\
\text { Porter des lunettes CE4 }\end{array}$ \\
\hline
\end{tabular}

\subsection{Pourquoi les $U V$ artificiels sont dangereux !}

Les conséquences à long terme des UV sont largement dépendantes des quantités reçues et de l'inégalité de sensibilité des phototypes cutanés. Les expositions, dans le cadre du travail en extérieur, représentent 4 à 8 fois les expositions «normales » des travailleurs postés (expositions quotidiennes extérieures et vacances) (ICNIRP, 2002, 1996).

Dans les pays «nordiques », les expositions aux UV artificiels pour le «bienêtre » sont très importantes et peuvent représenter 2 à 3 fois les UV solaires (ICNIRP, 2003). Une étude prospective (114 000 femmes), pratiquée dans les pays scandinaves, a montré les relations entre doses d'UV artificiels et mélanome malin cutané. Pour le public, en France, les UV artificiels utilisés à des fins cosmétiques sont encadrés par la législation (JO, 1997, décret 97-617). Actuellement, les recommandations de la directive européenne « basse tension » sont en conflit avec la norme IEC (2002) dans son évolution à partir de 2002. Un comité CEN ad hoc est chargé de régler le différent.

Les professionnels du bronzage observent, dans leur majorité, les recommandations de la législation française mais $20 \%$ n'en tiennent pas compte.

Dans le cadre du travail, les sources UV sont, en général, bien contrôlées mais les documents relatifs aux travailleurs, en extérieur ou postés ont tardé à paraître (ICNIRP/ILO/WHO, 2007). La médecine du travail n'est pas assez attentive à certaines conditions extrêmes d'exposition.

\section{Les recommandations de l'OMS pour l'Europe}

Le contrôle des sources de radiation UV devrait être une priorité de santé publique pour les autorités de santé nationales et locales. Le coût social et financier des 
cancers cutanés induits par les UV dans la population civile et chez les travailleurs (en extérieur) est important.

Les effets négatifs induits par les expositions aux radiations UV peuvent être évités dans une large mesure. Le but principal des efforts de prévention doit être dirigé vers une réduction des expositions aux RUV par une modification du comportement et de l'environnement.

Il convient d'utiliser largement l'index UV dans la communication et d'établir des Programmes d'éducation solaire dès le plus jeune âge. L'organisation d'espaces couverts dans les parcs, jardins, stades, écoles, et tous lieux publics, devrait être mieux prise en compte dans les travaux publics (WHO, 2003).

L'information des travailleurs d'extérieur et l'analyse de situation dans l'industrie utilisant les UV artificiels contribuerait certainement à une réduction des risques cutanés et oculaires.

\section{RÉFÉRENCES}

Chaney E.K., Sliney D.H. (2005) Re-evaluation of the ultraviolet hazard action spectrum - The impact of spectral bandwidth, Health Phys. 89, 322-332.

CIE (2002) DS 09.2/E Standard, Photobiological safety of lamps and lamp systems.

$19^{\mathrm{e}}$ directive (2006) Directive du Parlement Européen concernant les prescriptions minimales de sécurité et de santé relatives à l'exposition des travailleurs aux risques dus aux agents physiques (rayonnements optiques artificiels).

ICNIRP (1996) Guidelines on UV radiation exposure limits, Health Phys. 71, 78.

ICNIRP (1997) Guidelines on limits of exposure to broad-band incoherent optical radiation (0.38 to $3 \mu \mathrm{m})$, Health Phys. 77, 539-555.

ICNIRP (2002) General approach to protection against non-ionizing radiation, Health Phys. 82, 540548.

ICNIRP (2003) Health issues of ultraviolet tanning appliances used for cosmetic purposes, Health Phys. 84, 119-127.

ICNIRP/ILO/WHO (2007) Protecting workers from ultraviolet radiation.

IEC (2002) IEC 60335-2-27 International standard. Household and similar electrical appliances Safety - Part 2 -27: Particular requirements for appliances for skin exposure to ultraviolet and infrared radiation.

ISO/CIE (1998) ISO 17166/CIE S007, Erythema reference action spectrum and standard erythema dose.

JO (1997) JO RF Décret n 97-617 du 30 mai 1997 : décret relatif à la vente et à la mise à disposition du public de certains appareils de bronzage utilisant les rayonnements ultraviolets.

Kappes U.P. et al. (2006) Short and long-wave UV light (UVB \& UVA) induce similar mutations in human skin cells, J. Invest. Dermatol. 126, 667-675.

Mitchel D. (2006) Revisiting the photochemistry of solar UVA in human skin, PNAS 103, 1356713568.

Mouret S. et al. (2006) Cyclobutane pyrimidine dimers are predominant DNA lesions in whole human skin exposed to UVA radiation, PNAS 103, 13765-13770. 
Norme NF/EN 13032-1 (2004) Mesure et présentation des données photométriques des lampes et des luminances. Partie 1 : Mesurage et format des données.

Norme NF/EN 14255-1 à 4 (2005) Mesurage et évaluation de l'exposition des personnes aux rayonnements optiques incohérents. Partie 1 : rayonnements ultraviolets émis par des sources artificielles sur les lieux de travail (2005). Partie 2 : rayonnements visible et infrarouge émis par des sources artificielles sur les lieux de travail (2005). Partie 3 : rayonnements ultraviolets émis par le soleil (2007). Partie 4: terminologie et grandeurs utilisées pour le mesurage de l'exposition au rayonnement ultraviolet, visible et infrarouge (2006).

WHO (2003) UV radiation and health, Office régional pour l'Europe, Genève.

WHO/WMO/UNEP/ICNIRP (2002) Global solar UV-index. A practical guide.

WHO/UNEP/ICNIRP (1994) Ultraviolet radiation. Environmental health criteria 160, WHO Geneva.

Zuurbier M. et al. (2007) La santé environnementale des enfants en Europe: Priorités proposées par le réseau PINCHE. Environnement, Risques \& Santé 6, 43-56.

\section{Pour en savoir plus (ouvrages récents en français) et où trouver les documents}

Rayonnement Ultraviolet et Peau, F. Aubin, P. Humbert (Coord.). John Libbey, Eurotext, 2001, 268 pages.

Photodermatologie: Photobiologie cutanée, photoprotection et photothérapie. Société Française de Photodermatologie, Arnette Groupe Liaison, SA 2003, 377 pages.

Documents AFNOR : 11 rue Fancis de Pressensé, 93571 La Plaine Saint-Denis Cedex ; www.afnor.fr

Documents CIE/CNFE : 17 rue Hamelin, 75016 Paris, France.

Documents ICNIRP: Ingolstaedter Landst. 1, 85764 Oberschleissheim, Germany.

Documents IEC-CEN/CENELEC, UTE : 33 avenue du Général Leclerc, 92260 Fontenay-aux-Roses, France.

Documents OMS : 20 avenue Appia, CH-1211 Genève 27, Suisse. 